\title{
Screening of Precancerous Gastric Lesions by Serum Pepsinogen, Gastrin-17, Anti-Helicobacter Pylori and Anti- Caga Antibodies in Dyspeptic Patients over 50 years Old in Guilan Province, North of Iran
}

\author{
Fariborz Mansour-Ghanaei ${ }^{1 *}$, Farahnaz Joukar', Yaghoub Rajpout ${ }^{2}$, Tolou \\ Hasandokht $^{3}$
}

\begin{abstract}
Background: The aim of this study was to investigate the value of serum gastric markers to differentiate between patients with precancerous lesions and nonatrophic chronic gastritis. Materials and Methods: Serum samples of 128 patients with dyspepsia who were candidates for endoscopic examination were tested for pepsinogen (PG I and PG II), PG I/II ratio, gastrin 17(G-17), anti-Helicobacter pylori (anti-H pylori) and antiCagA antibodies. Two sample t-tests, chi-square tests and Pearson's correlation analyses were used for analysis using SPSS (version 20). Results: PGI, PG I/II ratio values were decreased significantly in the precancerous lesion group $(0.05,0.001$ respectively). The frequency of $H$ pylori infection was significantly $(p=0.03)$ different between the two groups ofthe study. Conclusions: We suggest PGI and the PG I/II ratio as valuable markers for screening of premalignant gastric lesions.
\end{abstract}

Keywords: Pepsinogen I - gastrin-17 - Helicobacter pylori antibodies - precancerous gastric lesion

Asian Pac J Cancer Prev, 15 (18), 7635-7638

\section{Introduction}

Gastric cancer (GC)is still highly prevalent in the world and considers as the second most common leading cause of cancer death (Parkin et al., 1992; Crew and Neugut, 2006; Yanaoka et al., 2008). GC is the fourth most common cancer worldwide, with 330, 290 to 603, 003 new cases among women and men respectively (Kamangar et al., 2006). Across continents, incidence rates reach to 26.9 per 100, 000 (Person Year) among males in Asia (Cunningham et al., 2005; Ries et al., 2008). Although, the incidence of stomach cancer has declined in more-developed regions (Munoz and Franceschi, 1997), it remains common in developing countries as a result of population aging, unhealthy lifestyle behavior such as smoking, sedentary life, and westernized diets (Munoz and Franceschi, 1997; Jemal et al., 2011). Iran, especially, the northern and northwestern regions are high risk areas for gastric cancer (Sajadi et al., 2005; Malekzadeh et al., 2009; Mansour-Ghanaei et al., 2012). Ardabil, as a northwestern province of Iran, has the highest incidence of gastric cancer (Sajadi et al., 2005). A strong spatial clustering of gastric cancer was found in both sexes in Mazandaran and Golestan, two provinces located on the Caspian Sea shoreline (Mohebbi et al., 2008).
Some experts in a 15 year trend analysis showed a steady pattern for gastric cancer in Guilan province. They also reported the age standardized incidence rate was 14.5 per $10^{5}$ (Atrkar-Roushan et al., 2013). The diagnosis of gastric cancer is usually late and thus, its prognosis is very poor (Maconi et al., 2008). In most areas of the world, except in Japan, Overall 5-year survival rates, estimate 20\% (Cunningham et al., 2005; Ries et al., 2008). Mass screening programs, staging systems in Japan, would help to achieve 5-year survival rates of approximately 60\% (Tsubono and Hisamichi, 2000; Parkin et al., 2002). In the diagnosis of atrophic gastritis and premalignant lesions, and also in the differentiation between healthy and diseased stomach mucosa, two options are available. Upper gastrointestinal (GI) endoscopy and histological examination of endoscopic biopsy is the first option. The second non-invasive option, is the examination of some serum markers, such as pepsinogen (PG I), pepsinogen I/II ratio (PG I/II ratio) and gastrin 17 (G-17) (Borch et al., 1989; Karnes Jr et al., 1991). Gastric PG I and PG II are produced by the chief and mucus neck cells in the gastric fundus. But, only, PG II is produced by the pyloric glands in the antrum and Brunner's glands.as a result of inflammation, both pepsinogen elevated and then, as atrophic gastritis progress the concentration of

${ }^{1}$ Gastrointestinal and Liver Diseases Research Center (GLDRC), ${ }^{3}$ Community Medicine Department, Guilan University of Medical Sciences, Rasht, ${ }^{2}$ Internal Medicine Department, Jahrom University of Medical Sciences, Jahrom, Iran *For correspondence: ghanaei@gums.ac.ir,ghanaie@yahoo.com 
PGII remains increased, while the concentration of PGI decreases. Consequently, the changes in pepsinogen may reflect the morphological status of the gastric mucosa (Foltmann, 1981).

In recent years, the usefulness of the serum PG I and PG I/II ratio, G-17 and H pylori antibodies (HpAb) has been investigated to early diagnose chronic gastric atrophy and for screening gastric cancer in some developed countries, including Europe and Japan (Sipponen et al., 2002; Vaananen et al., 2003; Ohata et al., 2004; Shiotani et al., 2005).

The aim of present study was to assess the serum tests: serum PGI, PG I/II ratio, G-17, H Pylori-IgG antibodies and Anti Cag A in precancerous lesions and nonatrophic chronic gastritis in patients with dyspepsia who had been diagnosed by Gastroscopy and biopsy.

\section{Materials and Methods}

We designed a cross sectional study to compare the serum gastric markers in precancerous lesions and nonatrophic chronic gastritis on 128 patients over 50 years old with dyspepsia between June 2013 to November 2013.The Medical Ethics Committee of Guilan University of Medical Sciences (GUMS) has approved the study design, protocols and informed consent procedure. This study was funded by Gastrointestinal and Liver Disease Research Center (GLDRC) of Guilan University of Medical Sciences.

Participants were recruited from GLDRC out patient clinic or private office. According to the inclusion and exclusion criteria, a total of 128 subjects with dyspepsia or epigastric pain who underwent endoscopy were divided into two groups based on endoscopic and histological findings: 40 patients in the precancerous (metaplasia, dysplasia, neoplasia and atrophic gastritis) group and 88 (including non atrophic gastritis) served as the control group. Patients over 50 years with any kind of dyspepsia willing to provide informed consent for study participation were included. Exclusion criteria were the following: history of gastric surgery, prior $H$ pylori eradication therapy, serious systemic disease such as Renal failure or liver disease, and those taking anti secretory or proton pump inhibitors four (4) weeks prior to the present study and anti-coagulant drugs or any condition which might have a negative effect on the compliance of the participants. Endoscopic and histological examinations before the serum tests, each subject underwent endoscopy. Subsequent two local anesthesia using Lidocaine $10 \%$ (with 10 minute interval) upper GI endoscopy (videoendoscope, GIF-Q240Z; Olympus Co., Tokyo, Japan) was performed by three experienced endoscopists and cooperation of a trained staff based on the standard guideline to decrease observer bias. Three samples were taken from different parts of stomach (body, fundus, antrum) and one sample from antrum for RUT to detect $H$ Pylori infection. The samples were fixed in formalin $10 \%$, labeled by subjects' codes and described by a blinded pathologist.

To decrease measurement bias, all blood samples were analyzed in a single private laboratory. The $5^{\mathrm{cc}}$ blood samples were taken after overnight fasting for measurement serum gastric markers.Determination of serum levels of PGI, PGII and G-17 was performed using a commercial ELISA assay (Gastropanel ${ }^{\circledR}$; $\left(\right.$ BioKit $^{\circledR}$, Helsinski, Finland), following the manufacturer's instructions. Anti-H Pylori IgG and anti-cag A antibody were analyzed by ELISA method ((Pishtaz teb Co. Iran and Diapro Kits) All descriptive statistics are presented as mean (standard deviation (SD)) for quantitative variables or number $(\%)$, for categorical variables. To compare total premalignant lesion with nonatrophic gastritis, we created a new group named precancerous lesion consist of metaplasia, dysplasia, neoplasia and atrophy.

Two-sample t-tests were used for the comparison of continuous variables and Pearson's chi-square tests were used for the comparison of categorical variables. The Pearson correlation analyses were used to study the bivariate relationships between serum gastric markers and gastric lesions.Statistical analysis was carried out with the SPSS (version 20) and $p<0.05$ was considered statistically significant.

\section{Results}

A total of 128 patients (71 women, 55\%) with an average age of 66.5 years were included. The two most common symptoms were epigastric pain and dyspepsia. Endoscopy was compatible with nonatrophic chronic gastritis in 120 patients, showed dysplasia in 5 patients, metaplasia in 39 patients and neoplasia in 6 patients. $H$ pylori infection based on the serologic test was positive in $55 \%$ (71) of participants. The distribution of nonatrophic chronic gastritis and precancerous lesions in both sexes were similar (Table 1).

The percentage of low, normal and high serum PGI and PGI/PGII ratio were significantly different in patients with precancerous lesions to chronic gastritis $(\mathrm{p}=0.005$, 0.03 , respectively). Furthermore, the frequency of positive $H$ pylori $\mathrm{IgG}$ patient was significantly $(\mathrm{p}=0.03)$ different between chronic gastritis and precancerous lesion groups (Table 2).

The mean value of PGI and PGI/PGII ratio were

Table 1. Distribution of Demographic Data

\begin{tabular}{lrcrrr}
\hline Variable & Precancerous lesion & Chronic gastritis & p value \\
\hline Sex (women) no. (\%) & 18 & $(45)$ & 53 & $(60.2)$ & 0.10 \\
Age (mean, SD) & 68 & $(9.5)$ & 65 & $(9.2)$ & 0.09 \\
History of smoking & 4 & $(10)$ & 10 & $(11.4)$ & 0.80 \\
History of alcohol & 0 & & 1 & $(1.1)$ & 0.40 \\
\hline
\end{tabular}

Table 3. Levels of Serum Gastric Markers in Different Groups

\begin{tabular}{lcrrl}
\hline & $\begin{array}{c}\text { Precancerous } \\
\text { Mean(SD) }\end{array}$ & $\begin{array}{c}\text { Chronic gastritis } \\
\text { Mean(SD) }\end{array}$ & p \\
\hline PGI & $99.5(76.8)$ & $100.2(62.8)$ & 0.05 \\
PGII & $20 \quad(17.3)$ & $17.3(11.5)$ & 0.3 \\
PGI/ PGII & $5.8(3.2)$ & $8.7(4.4)$ & 0.001 \\
G-17 & $49.4(84.8)$ & $30.5(37.6)$ & 0.8 \\
Anti H pylori IgG & $40.5(24.7)$ & $42.3(23.5)$ & 0.6 \\
Anti CagA Ab & $27.3(35.2)$ & $19.3(28.2)$ & 0.1 \\
\hline
\end{tabular}


Table 2. Distribution of Serum Gastric Markers in Precancerous Lesion and Chronic Gastritis

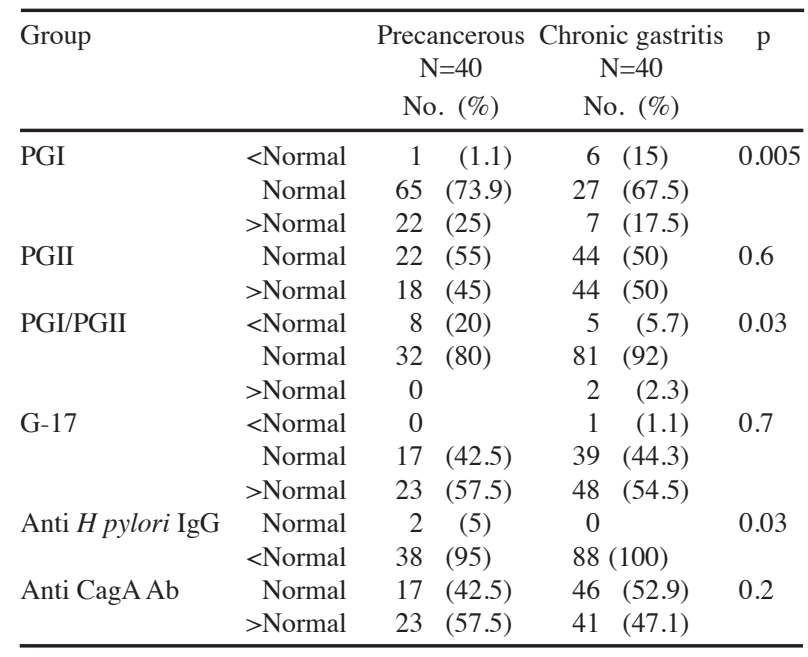

significantly different in patients with precancerous lesions to nonatrophic chronic gastritis $(\mathrm{p}=0.05,0.001$, respectively). There was no statistically significant difference in the mean value of PGII, G-17 and anti CagA between precancerous lesion and non atrophic gastritis (Table 3).

Bivariate relationships were determined between some serum gastric marker and gastric lesions. PGI and PGI/ PGII ratio was significantly correlated with precancerous lesions $(\mathrm{p}<0.05)$. There was no significant correlation between

\section{Discussion}

The results of our study showed that the frequency of reduced PGI and PGI/PGII ratio was more common in patients with precancerous lesions. Those who had any of the premalignant histologic gastric lesions (metaplasia, neoplasia and dysplasia) have been reported the lower mean value of PGI and PGI/PGII. Ratio, urthermore, we detected the mean value of PGI and PGI/PGII ratio were significantly lower in patients with precancerous lesion than nonatrophic gastritis. This finding coheres with the results of a large-scaled study in the hot point regions of Iran for GC designed to assess prognostic value of screening biomarkers like pepsinogen I/II and G17 (Shafaghi et al., 2012). In this study, Shafaghi et al present the cut off value of $\mathrm{PGI}<90.6 \mathrm{ng} / \mathrm{ml}$ with the sensitivity and specificity of $64.7 \%$ and $40 \%$ respectively for any of three lesions (metaplasia, dysplasia or atrophy). Qin CAO et al described serum PGI and PGI/PGII level decreased in atrophic gastritis and gastric cancer groups. In a 10 years follow up of 5, 209 asymptomatic subjects, Yanaoka et al. (2008) showed gastric cancer risk increased with a reduced serum PG I level or a reduced PG I/II ratio.

In our study, the serum gastrin level were similar in patients with precancerous lesions and nonatrophic chronic gastritis. Sheykholeslami et al find neither PGI, the PG I:II ratio, gastrin 17, nor their combination were able to predict precancerous conditions among the first-degree relatives of gastric cancer patients (Haj-sheykholeslami et al., 2008). Similarly, a case control study in Peru reported serum gastrin-17 can not be considered as a useful screening marker in early detection of gastric cancer (Colarossi et al., 2010). On the other hands, some previous researches showed that low levels of serum G-17 in combination with low serum PGI or PGI/PGII ratio identifies patients with advanced and extensive atrophic gastritis in both the antrum and corpus (Karnes Jr et al., 1991; Vaananen et al., 2003). While, in other research, the increased serum Gastrin was found to be as a marker for advanced corpus atrophy (Sipponen et al., 2002). Furthermore, Shafaghi et al. (2012) reported the serum G-17 might be a good biomarker for gastric atrophy or metaplasia. In this area, Zhang et al. (2007) described in a large sample mass screening study, the serum gastrin level can be elevated in the precancerous stage of gastric cancer, but can be significantly reduced on the onset of the formation of gastric cancer. Some probable reasons for this discrepancy are: the serum Gastrin content can be influenced by many factors, such as age, lesion sites, disease types and H Pylori infection.

Our study indicated that the frequency of H pylori IgG were different between chronic gastritis and precancerous lesions groups. This finding was confirmed by some previous researches which have been reported serum anti $H$ pylori and pepsinogens were found to be useful in distinguishing subjects with non-atrophic gastritis from those with normal mucosa (Vaananen et al., 2003; Germana et al., 2005). Another studyshowed H Pylori can increase the serum gastrin level; and the elevation of serum gastrin level by $H$ Pylori may be one of its carcinogenic mechanisms (Gisbert et al., 1996).

In our study, the frequency of anti cag A was similar in precancerous and chronic gastritis groups. Although, Backert et al. (2009) indicated a significant association of cag PAI presence with histopathological findings in gastritis, peptic ulcer, and gastric cancer patients. Recently, researcher found that, the cag PAI appeared not to be useful predictor for $H$ Pylori-related diseases in Iranian patients (Baghaei et al., 2009; Jafarzadeh et al., 2009).

An important aspect of the current study refers to compare the serum gastric markers in precancerous patients with nonatrophic chronic gastritis. While, majority of previous studies tried to assess the gastric biomarkers ability to predict gastric cancer or to compare serum gastric level between healthy people and patients with precancerous lesions or gastric cancer. Our result confirmed our previous finding suggested serum PGI and PGI/II ratio screening can detect premalignant lesions (Shafaghi et al., 2012).

Our study, while having much strength, involved a limitation that should be considered. We can not assess the serum gastric markers in two groups of study regard to location of the lesion because of small sample size in each group. So, further research with larger sample size in every group were required.

In conclusion, analyses of serum PGI and PGI/II ratio offer important information regarding the presence of premalignant gastritis lesions and provide a useful marker for screening gastric disease. Serum pepsinogen could be recommended as a first step to dyspeptic patients who have no alarming symptoms. 


\section{Acknowledgements}

This study was supported by GLDRC of Guilan University of Medical Sciences. The authors' heartfelt thanks are extended to all members of GLDRC, endoscopy center and all the patients who so kindly agreed to participate in this study. This study is a part of a subspecialty thesis. There was no competing interest to declare.

\section{References}

Atrkar-Roushan Z, Kazemnejad A, Mansour-Ghanaei F, Zayeri F (2013). Trend analysis of gastrointestinal cancer incidences in Guilan province: comparing rates over 15 years. Asian Pac J Cancer Prev, 14, 7587-93.

Backert S, Schwarz T, Miehlke S, et al (2004). Functional analysis of the Cag pathogenicity Island in Helicobacter pylori isolates from patients with gastritis, peptic ulcer, and gastric cancer. Infect Immun, 72, 1043-56.

Baghaei K, Shokrzadeh L, Jafari F, et al (2009). Determination of Helicobacter pylori virulence by analysis of the Cag pathogenicity island isolated from Iranian patients. Dig Liver Dis, 41, 634-8.

Borch K, Axelsson C, Halgreen H, et al (1989). The ratio of pepsinogen A to pepsinogen C: a sensitive test for atrophic gastritis. Scand J Gastroenterol, 24, 870-6.

Colarossi A, Inga R, Prochazka R, et al (2010). Pepsinogen and gastrin in the noninvasive diagnosis of gastric atrophy. A case-control study in Peruvian population. Rev Gastroenterol Peru, 31, 110-5 (in Spanish).

Cunmingham S. C, Kamangar F, Kim M. P, et al (2005). Survival after gastric adenocarcinoma resection: eighteenyear experience at A single institution. J Gastrointest Surg, 9, 718-25.

Foltmann B(1981). Gastric proteinases-structure, function, evolution and mechanism of action. Essays Biochem, 17, 52-84.

Germana B, Dimario F, Cavallaro L, et al (2005). Clinical usefulness of serum pepsinogens I and II, gastrin-17 and anti-helicobacter pylori antibodies in the management of dyspeptic patients in primary care. Dig Liver Dis, 37, 501-8.

Gisbert J, Boixeda D, Vila T, et al (1996). Helicobacter pylori infection and basal levels of serum gastrins in patients with duodenal ulcer and subjects with normal endoscopy. Med Clin, 106, 325-8 (in Spanish).

Haj-Sheykholeslami A, Rakhshani N, Amirzargar A, et al (2008). Serum pepsinogen I, pepsinogen Ii, and gastrin 17 in relatives of gastric cancer patients: comparative study with type and severity of gastritis. Clin Gastroenterol Hepatol, 6, 174-9.

Jafarzadeh A, Hassanshahi G, Nemati M (2009). Serum levels of high-sensitivity C-reactive protein (Hs-Crp) in Helicobacter pylori-infected peptic ulcer patients and its association with bacterial CagA virulence factor. Dig Dis Sci, 54, 2612-6.

Jemal A, Bray F, Center M. M, et al (2011). Global cancer statistics. CA Cancer J Clin, 61, 69-90.

Kamangar F, Dores GM,Anderson WF (2006). Patterns of cancer incidence, mortality, and prevalence across five continents: defining priorities to reduce cancer disparities in different geographic regions of the world. J Clin Oncol, 24, 2137-50.

Karnes Jr W, Samloff I, Siurala M, et al (1991). Positive serum antibody and negative tissue staining for Helicobacter pylori in subjects with atrophic body gastritis. Gastroenterology, 101, 167-74.

Maconi G, Manes G, Porro GB (2008). Role of symptoms in diagnosis and outcome of gastric cancer. World $J$
Gastroenterol, 14, 1149-55.

Malekzadeh R, Derakhshan M. H, Malekzadeh Z (2009). Gastric cancer in Iran: epidemiology and risk factors. Arch Iran Med, 12, 576-83.

Mansour-Ghanaei F, Joukar F, Soati F, Mansour-Ghanaei A, Naserani SB (2012). Knowledge about gastric carcinoma in north of Iran, a high prevalent region for gastric carcinoma: a population-based telephone survey. Asian Pac J Cancer Prev, 13, 3361-66.

Mohebbi M, Mahmoodi M, Wolfe R, et al (2008). Geographical spread of gastrointestinal tract cancer incidence in the Caspian sea region of Iran: spatial analysis of cancer registry data. Bmc Cancer, $8,137$.

Munoz N, Franceschi S(1997). Epidemiology of gastric cancer and perspectives for prevention. Salud Publica Mex, 39, 318-30.

Ohata H, Kitauchi S, Yoshimura N, et al (2004). Progression of chronic atrophic gastritis associated with Helicobacter pylori infection increases risk of gastric cancer. Int J Cancer, 109, 138-43.

Parkin D, Whelan S, Ferlay J, Teppo L, Thomas DB (2002). Cancer incidence in five continents vol. Viii. Iarc Scientific Publications, 155.

Ries L, Melbert D, Krapcho M, et al (2008). Seer cancer statistics review, 1975-2005. Bethesda, Md: National Cancer Institute, 1975-2005.

Sajadi A, Nouraie A, Mohagheghi M, et al (2005). Cancer occurrence in Iran in 2002, an international perspective. Asian Pac J Cancer Prev, 6, 359-63.

Shafaghi A, Mansour-Ghanaei F, Joukar F, et al (2012). Serum gastrin and the pepsinogen I/II ratio as markers for diagnosis of premalignant gastric lesions. Asian Pac J Cancer Prev, 14, 3931-6.

Shiotani A, Iishi H, Uedo N, et al (2005). Histologic and serum risk markers for noncardia early gastric cancer. Int J Cancer, 115, 463-9.

Sipponen P, Ranta P, Helske T, et al (2002). Atrophic gastritis serum levels of amidated gastrin-17 and pepsinogen I in atrophic gastritis: an observational case-control study. Scand J Gastroenterol, 37, 785-91.

Tsubono Y, Hisamichi S (2000). Screening for gastric cancer in Japan. Gastric Cancer, 3, 9-18.

Vaananen H, Vauhkonen M, Helske T, et al (2003). Nonendoscopic diagnosis of atrophic gastritis with a blood test. Correlation between gastric histology and serum levels of gastrin-17 and pepsinogen I: a multicentre study. Eur $J$ Gastroenterol Hepatol, 15, 885-91.

Yanaoka K, Oka M, Yoshimura N, et al(2008). Risk of gastric cancer in asymptomatic, middle-aged Japanese subjects based on serum pepsinogen and Helicobacter pylori antibody levels. Int J Cancer, 123, 917-26.

Zhang Z, Gong Y. H, Wang X. G, et al (2007). Factors affecting the serum gastrin 17 level: an evidence-based analysis of 3906 serum samples among Chinese. J Dig Dis, 8, 72-6 